\title{
Standing wave-mediated molecular reorientation and spontaneous formation of tunable, concentric defect arrays in liquid crystal cells
}

\author{
L K Migara and Jang-Kun Song
}

Standing waves are involved in various fundamental, natural phenomena over a wide range of scales, that is, from electron orbitals to planetary oscillations. Interactions between standing waves and matter are of interest. Here we demonstrate spontaneous, nematodynamic standing waves in a nonlinear liquid crystal medium and their interactions with director fields. The standing waves hold unique features such as diagonal nodal lines, self-adaption to broad resonance frequencies and a step-like increase in the wavelength with the increasing frequency. Furthermore, the standing wave causes unexpected phenomena that can be used in various applications; for example, it can manipulate the molecular orientation to form tunable, periodic defect arrays with concentric director profiles, which can serve as an optical vortex array inducer or tunable micro-liquid crystal lens array. The standing wave also exerts asymmetric mechanical pressure that can relocate small particles. The results lead to new approaches in director manipulation, colloidal assembly and singular optics.

NPG Asia Materials (2018) 10, e459; doi:10.1038/am.2017.217; published online 5 January 2018

\section{INTRODUCTION}

Standing waves and their interactions with matter constitute the basis of modern physics, and they are involved in various fundamental phenomena over a wide range of scales. ${ }^{1,2}$ The De Broglie standing wave explains the origin of matter formation, that is, it stabilizes electronic orbitals and generates an electronic bandgap in matter. Electromagnetic standing waves also cause various fundamental phenomena, for example, particle trapping, ${ }^{3}$ tuning electron density profiles $^{1}$ and lasing. Mechanical standing waves are involved in the heat capacity and superconductivity of matter on the nanoscale and can be observed in massive, wobbly bridges on the macroscale. Recently, the use of mechanical standing waves to relocate microparticles on Faraday waves and in lab-on-a-chip technology has attracted interest, ${ }^{4-6}$ but using their interactions for molecular ordering in liquid or soft matter has not been explored.

In this study, we demonstrate spontaneous standing nematodynamic waves in liquid crystals (LCs) within cavity and their molecular ordering interactions. In contrast to conventional waves, $, 7,8$ a standing nematodynamic wave in a LC-filled thin cavity has several unexpected properties, such as self-adaption to broad resonance frequencies, a step-like increase in the wavelength with the increasing frequency, only even modes, and diagonal nodal lines. The wave interactions with the LC director ordering is particularly interesting; the wave can rearrange the orientations of the LCs to form concentric defect arrays with left and right helicities, that is, arrays of chiral umbilic defects.

In fact, pattern generation is a popular topic in LCs and can be implemented via various methods, such as self-assembly, ${ }^{9}$ confinement effect, ${ }^{10}$ defect-assisted assembly, ${ }^{11}$ electrohydrodynamic convection, ${ }^{12}$ ionic effect, ${ }^{13}$ surface effects ${ }^{14}$ and dielectrophoresis ${ }^{15}$; however, standing nematodynamic waves have not been used. Additionally, tunable concentric defect arrays have not been achieved via another method. Well-controlled defects in LCs can serve as optical vortex inductors, ${ }^{16-20}$ which have recently attracted attention for their fundamental properties and potential applications in quantum computational schemes and large data optical communication. $^{21,22}$

\section{MATERIALS AND METHODS}

LC cells were prepared by sandwiching two glass substrates. The glass substrates contained square indium tin-oxide electrodes with dimensions of $2 \times 2 \mathrm{~cm}^{2}$. A commercial, vertical-alignment polyimide solution, AL60101 (JSR Company, Tokyo, Japan), was coated on the substrate, and the substrate was baked at $230^{\circ} \mathrm{C}$ for $1 \mathrm{~h}$. The substrate was not treated by a rubbing process. The $3.5 \mu \mathrm{m}-$ thick cell cavity was sustained by bead spacers and was filled with a commercial nematic LC mixture, MLC7026 (Merck Co., Korea), with a negative dielectric anisotropy $(\Delta \varepsilon=-3.9)$. For the chiral dopant, we used R811 or S811 (Merck Co., Korea) with a helical twisting power of $\sim 11 \mu \mathrm{m}^{-1}$. A $0.2 \mathrm{wt} \% \mathrm{R} 811$ (or S811) solution was added to MLC7026, and the mixture was heated above the isotropic phase transition temperature and cooled down to achieve homogeneous mixing.

For fluorescence confocal polarizing microscopic (FCPM) observations, ${ }^{23,24}$ a $0.01 \mathrm{wt} \%$ coumarin-6 (C6) solution of dye molecules was added into the LC cell, and a laser scanning-type FCPM (K1-Fluo, Nanoscope systems, Korea) instrument was used to obtain the fluorescence images. The C6 molecules align 
along the LC director, and the fluorescence images can reveal the LC aligning direction.

To demonstrate the particle actuation, we added $2 \mathrm{wt} \%$ of the C6 dye to the LC cell. The $2 \mathrm{wt} \% \mathrm{C} 6$ solution dispersed well at $50^{\circ} \mathrm{C}$, but after a certain time at room temperature, the $\mathrm{C} 6$ molecules aggregated to form micrometer-sized rods. The crystallized C6 rods were uniformly distributed throughout the LC cell. The size and density could be tuned by controlling the C6 concentration and storage time at room temperature.

\section{RESULTS}

Pulse-train signal-induced periodic defect array in a LC cell Sound waves and mechanical waves can affect the ordering of molecules in LCs. ${ }^{25-27}$ However, the manipulation of molecular ordering using controlled pressure waves or standing waves has been unexplored because of the difficulty in precisely controlling the manipulation. We devised a simple method to generate a standing nematodynamic wave and induced the interaction between the standing wave and molecular order using a vertically aligned LC cell containing LCs with a negative dielectric anisotropy. When a squarewave electric field was applied across the LC layer, the cell exhibited a random umbilical texture, as shown in Figure 1a and its expanded image in the bottom. This indicates that the molecular tilting did not have a preferred direction under a square-wave electric field. In contrast, when a pulse-train signal was applied (top panel in Figure 1b), two-dimensional (2D) periodic patterns with varying pitches (wavelength) were spontaneously formed, as shown in Figure $1 \mathrm{~b}$. The rotation of the polarizers yielded an inversion of the brightness profile (inset image), which revealed the spatial variation in the director tilting direction.

The observations of the microscopic organization at low frequencies $(<20 \mathrm{~Hz})$ revealed numerous microdomains with irregular shapes and oppositely oriented directors divided by double-wall boundaries (Figure 2a). The domain shapes changed whenever a pulse signal was refreshed at low frequencies, but as the frequency increased $(>20 \mathrm{~Hz}$ ), the microdomains merged to form two types of macroscopic domains that did not change their shapes with the refreshing pulse signals, as shown in Figure 2b. Depending on the viewing direction and polarizer

a

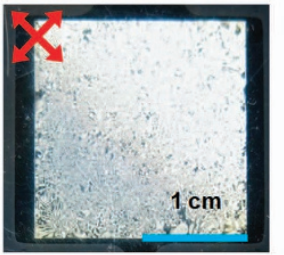

b
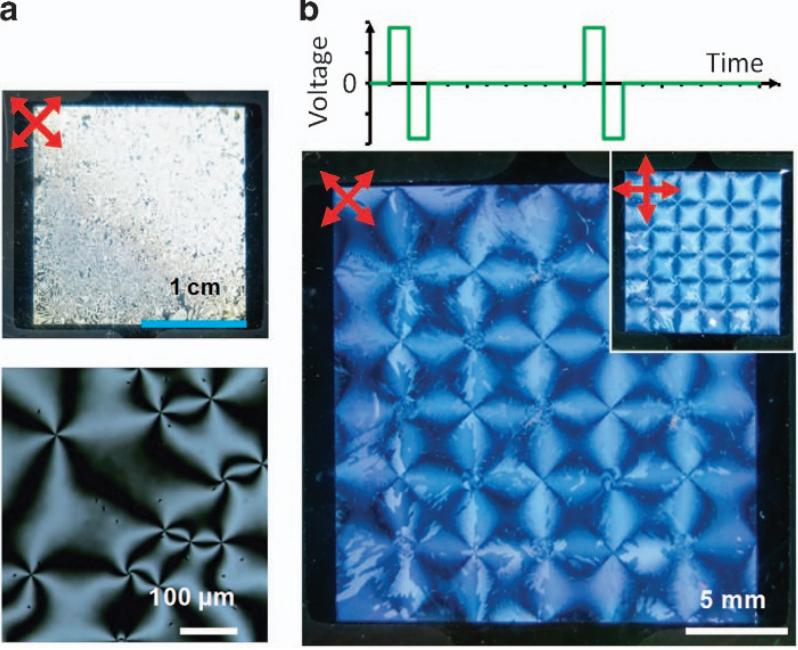

Figure 1 Periodic patterns under a pulse-train signal. (a) In the presence of a square-wave field, a vertical alignment cell exhibited a random texture with umbilical defects owing to the absence of an easy axis. (b) In contrast, in response to a train of pulses $(10 \mathrm{~V}$ and $20 \mathrm{~Hz}$ ), a blinking periodic pattern appeared. When the crossed polarizers were rotated (arrows), dark areas became bright and vice versa (inset). direction, the luminance of each domain varied owing to the molecular orientation, which allowed us to accurately determine the mean director direction at any position in the cell (see Supplementary Figure 1 for the details of the experiment). When a unit pattern is obliquely observed, the two domains exhibit a clear contrast, as illustrated in Figure 2b, and the white and brown arrows indicate the molecular tilting directions (see the inset illustration). The molecular orientations are opposite in the neighboring macroscopic domains in each wing of a periodic unit. In this way, we experimentally determined the mean director profile for the entire area of the cell. The periodic pattern was composed of defect arrays of +1 defects (solid circles and chiral umbilics) and -1 defects (open circles), as described in Figure 2c, in which the double-sided arrows indicate the director direction with the oppositely tilted domains. Interestingly, the director profile around the +1 defects has a concentric arrangement, that is, a vortex structure. ${ }^{16}$ Although the +1 defects with radial director profiles are frequently found in LC cells, ${ }^{13}$ the concentric director profiles are rarely observed. To reconfirm the director field structure, we examined the director profiles near the +1 and -1 defects using two methods, an optical compensator technique and an FCPM analysis. As shown in Figure 2d, when a quarter-wave plate (QWP) was inserted between the cell and the analyzer, the colors of the areas with directors parallel and perpendicular to the QWP axis became yellowish and bluish, respectively. Based on the color distribution, the director profiles were determined, as shown in Figure $2 \mathrm{~d}$, which is the same as those in Figure 2c. In the FCPM analysis shown in Figure 2e, the brightest areas in the fluorescence image have a director field parallel to the polarizer axis. ${ }^{23}$ By rotating the polarizer, the concentric profiles near the +1 defect and the tetraparabolic profile near the -1 defects were analyzed, as shown in Figures $2 \mathrm{e}-\mathrm{i}$ and $\mathrm{e}-\mathrm{ii}$, respectively, and agree well with those in Figures $2 \mathrm{c}$ and $\mathrm{d}$.

Figure $2 \mathrm{f}$ is the area enclosed by the yellow box in Figure 2c. Both domains in the center area appear dark under the diagonally crossed polarizers (the top panel in Figure 2f), indicating that the mean directors align along the diagonal direction in both domains. However, interestingly, when rotating the analyzer (yellow arrow) in an anticlockwise direction, domain A becomes darker and domain B becomes bright, as indicated in the bottom panel in Figure $2 \mathrm{f}$. When rotating in a clockwise direction, an opposite luminance change is observed. This clearly indicates that the two domains have opposite twist deformations with the same mean director directions.

Array patterns of splay umbilical defects with radial director fields were recently reported using ionic modulation near a polymer interface, and the effect is related to electric actions on ions in a LC medium. ${ }^{13}$ The LCs in their experiment contain large amounts of ionic compounds, and this effect cannot be the origin of the pattern formation in our study using pure LCs without ions (Supplementary Figure 2). To verify the lack of connection between the periodic pattern and the ionic effect, we redesigned the pulse-train signal to have a higher frequency within the pulse period, as shown in Figure 3, where the $\mathrm{S}_{\mathrm{L}}$ and $\mathrm{S}_{\mathrm{H}}$ signals are the standard and modified pulse-train signals, respectively. The overall pulse frequency is $22 \mathrm{~Hz}$ in both cases, and the frequencies of the signals within a pulse are $458 \mathrm{~Hz}$ and $3.7 \mathrm{kHz}$ for $\mathrm{S}_{\mathrm{L}}$ and $\mathrm{S}_{\mathrm{H}}$, respectively. A typical field-induced response of LCs based on an induced polarization follows the quadrupolar response, and so, both the $\mathrm{S}_{\mathrm{L}}$ and $\mathrm{S}_{\mathrm{H}}$ signals result in the same director switching. However, the dynamics of the ions that are subjected to the dipolar response to electric fields are dependent on the frequencies of the signals. Hence, the ionic convection should be significantly attenuated at higher frequencies. When switching the 

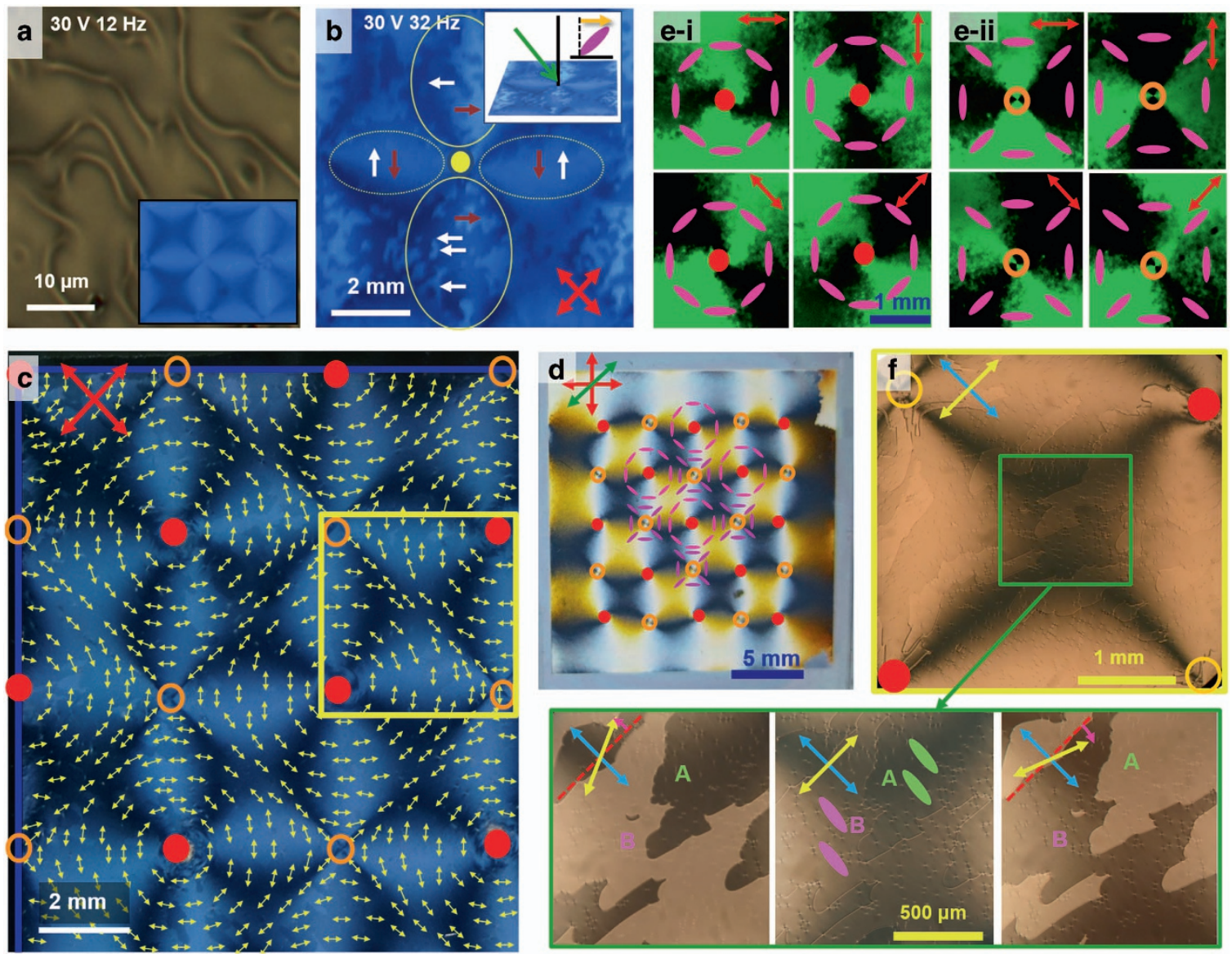

Figure 2 Defect array with concentric director fields and verifications. (a) Microdomains appeared at low frequencies ( $<20 \mathrm{~Hz}$ ). These were not macroscopically discernible (inset). (b) At frequencies above $20 \mathrm{~Hz}$, the microdomains merged into larger macroscopic domains, which were discernible on oblique observation. c The director fields profile: double-headed arrows denote the degenerated director fields with oppositely oriented domains. Red solid and orange open circles are +1 and -1 defects. (d and $\mathbf{e}$ ) The director profiles around the defects were reconfirmed using an optical compensator (e) and FCPM observations (e). The red arrows denote the polarizers, and the green arrow is the direction of the quarter-wave plate. Purple ellipses are the director alignment. S811 was added for the cells used in (d-f). The dark area enclosed by four defects under the diagonally crossed polarizers. When rotating the analyzer anti-clockwise or clockwise, the contrast between the two domains inversely changed, which indicated that the two domains have opposite twist deformations.

signal from $\mathrm{S}_{\mathrm{L}}$ to $\mathrm{S}_{\mathrm{H}}$ or vice versa, the periodic pattern in the cell remained unchanged, as shown in the bottom images in Figure 3. Thus, the ionic effect was ruled out. When ions were intentionally added into our cell, an electrohydrodynamic convective pattern can partially coexist under a certain condition (Supplementary Figure 3). This also supports the idea that the standing wave pattern is not related to the electrohydrodynamic convection.

\section{Pressure standing waves and director reorientation}

The addition of small amount of a chiral dopant into a LC readily eliminated the oppositely oriented domains, and none of the microdomains at low frequency or macroscopic domains at high frequency were observed. (This will be further discussed in the next section.) To verify that the concentric director profile can be induced by pressure waves, we periodically pressed a cell containing a chiral dopant-added LC using the tip of a pair of tweezers to generate outward and inward periodic LC flows near the tip while simultaneously applying a squarewave electric field. As shown in Figure 4a, the periodic LC flows produced $a+1$ defect with the same concentric director fields around the pressure point. The director direction indicates the tilting direction from the vertical axis (see the inset in Figure 4a). The concentric director profile at the moment of the pressing was anti-clockwise, which indicated that the molecules tilted in the anti-clockwise direction. However, upon releasing the pressure, the tilting direction was clockwise, as shown by the purple arrows in Figure 4a. The reversal of the director vortex handedness was confirmed by the reversed brightness in the images. The molecular orientation was perpendicular to the LC flow direction. In addition, the periodic defect array with concentric director profiles was imitated by imposing an external pressure at multiple, appropriate defect points, as shown in Figure $4 \mathrm{~b}$. Pressing four points with the tips of two tweezers induced spreading flow from the pressure points and redirected the molecular tilting direction to form +1 defect arrays with the same concentric director fields as those in Figure 2c. Additional defect points were automatically generated throughout the cell (red points in Figure $4 \mathrm{~b}$ ), which suggested interferences from the pressure waves that were reflected from the boundaries. Moreover, the directors around the pressing points had an anti-clockwise director tilting arrangement, while those in the automatically generated defect points had an opposite director tilting arrangement. This indicated that the automatically generated points have a converging LC flow. When releasing the pressure, the handedness of the concentric director profile was 

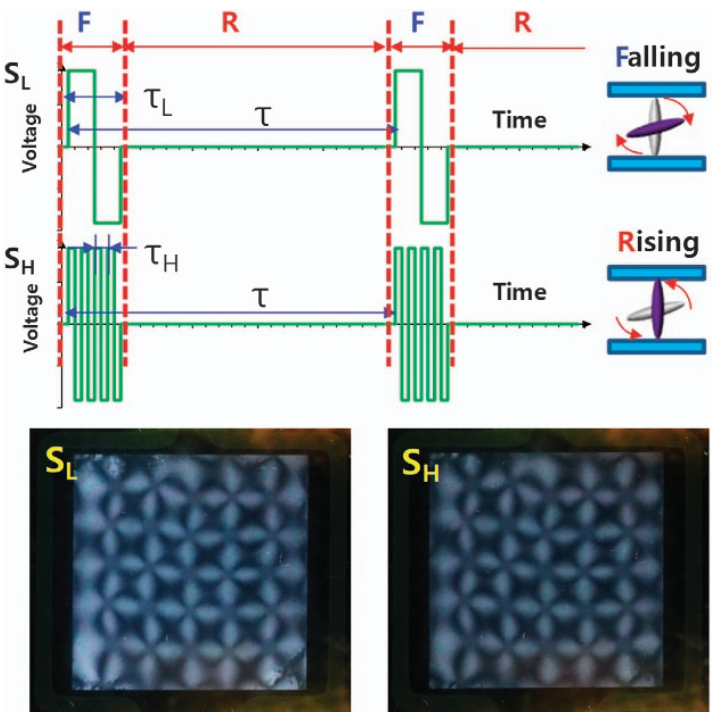

Figure 3 Quadrupolar response of the periodic pattern to electric fields. When the signal is switched from $S_{L}$ to $S_{H}$ (top), no change was observed in the periodic pattern of the cell (bottom). $\tau=45 \mathrm{~ms}\left(f_{\tau}=22 \mathrm{~Hz}\right), \tau_{\mathrm{L}}=2.2 \mathrm{~ms}$ $\left(f_{\tau L}=458 \mathrm{~Hz}\right)$, and $\tau_{\mathrm{H}}=0.27 \mathrm{~ms}\left(f_{\tau \mathrm{H}}=3.7 \mathrm{kHz}\right)$. This indicates that the periodic pattern is not related to ionic action under the application of electric fields.
Flow-induced temporal pretilt and twisted director deformation under an electric field application

The radial flow around the pressing point may induce a bend deformation in the LC layer, resulting in a slight molecular tilting near the surface (left in Figure 5a), which is different from a purely vertical alignment. Then, the application of an electric field to the cell with a flow-induced pretilt angle will cause the LC molecules to rearrange to have a minimum total free energy, including the electrical energy and elastic deformation energy. A computer simulation using a commercial LC software (Techwiz, Sanayi Systems Company, Korea) revealed that the LC layer can have either a left- or right-twist deformation with a mean director normal to the flow direction in the middle layer, as described in Figure 5a. (The details of the simulation method are explained in Supplementary Figure 4.) As shown in Figure $5 \mathrm{~b}$, the mean azimuthal angles in the left- and right-twist deformations are $90^{\circ}$ and $-90^{\circ}$, respectively, and the two deformations have the same total free energy. The flow-induced twist deformation was experimentally confirmed, as shown in Figure $2 \mathrm{f}$. The addition of a chiral dopant breaks the symmetry between the two opposite twist deformations (Supplementary Figure 4), and only a single type of twisting domain is obtained. It is interesting to note that the mean directors align in a manner normal to the flow direction. The illustrations in Figure $5 \mathrm{c}$ show the possible director deformations under the application of different electric fields. Owing to the initial
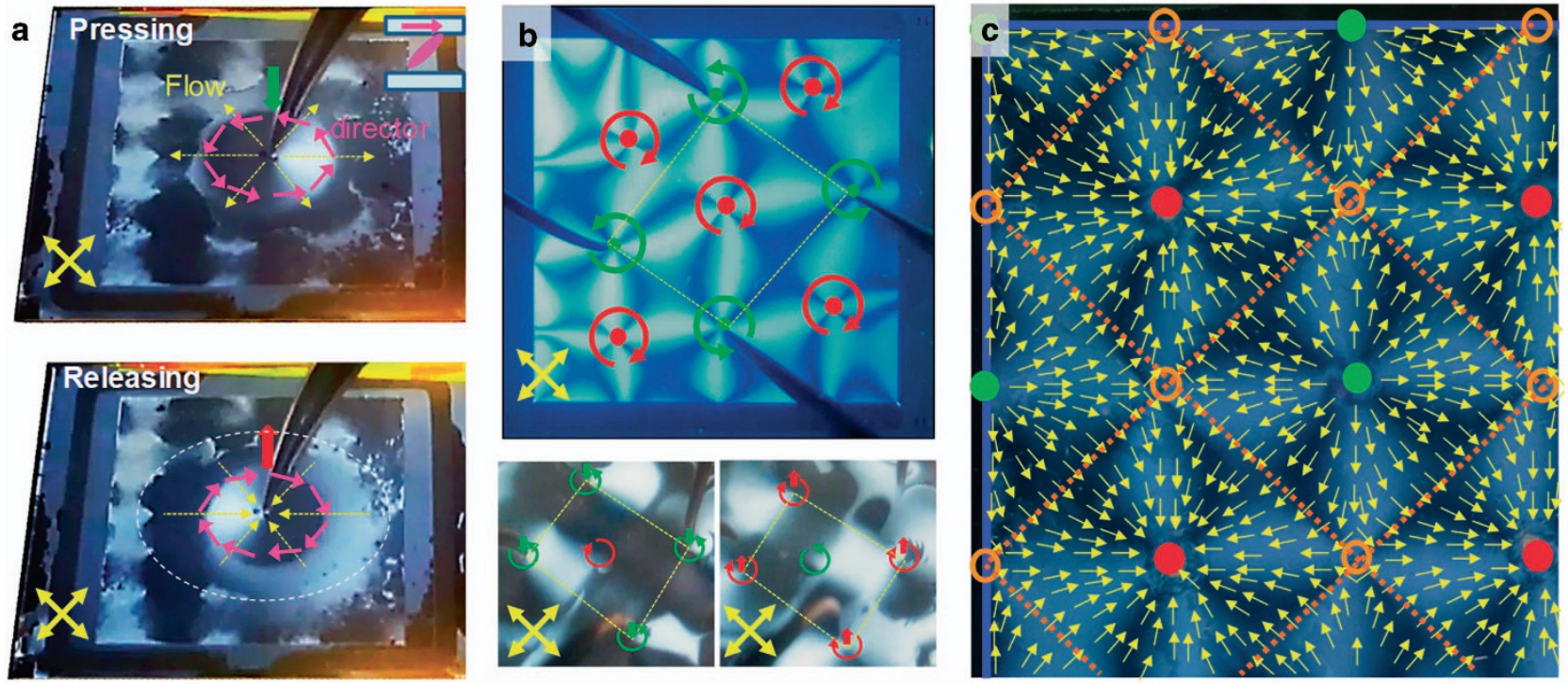

Figure 4 Defect array imitated by external pressure waves and coupling between the pressure wave and director profile. (a) By pressing a cell with a sharp tweezer tip, an outward LC flow generated an anti-clockwise circular director profile (top). In contrast, upon releasing the cell, an inward LC flow produced a clockwise director arrangement (bottom). A 0.2 wt\% R811 chiral dopant was added to the LC. (b) Pressing four points with sharp tweezer tips produced a similar pattern with the same +1 defect as that in Figure $1 \mathrm{~b}$. The bottom images, which were obtained from an oblique observation, exhibit the inversion of the handedness of the concentric director profiles of both the pressing points and the automatically generated defect point (center point). Red and green circular arrows denote the clockwise and anti-clockwise director arrangements. (c) Flow vector fields at the 'pulse ON' time ( $p_{+}$stage); the red and green solid circles denote the converging and diverging flow points (that is, the highest and lowest pressure points), respectively. The dotted lines are nodal lines.

inversed, which is indicated in the photos of the central part of the cell (the bottom of Figure $4 \mathrm{~b}$ ) that were taken from the oblique direction. Based on the relationship between the LC flow and director profile that was discovered in this experiment, the flow vector fields in the cell under the pulse-train signal can be constructed as shown in Figure 4c. Thus, it is apparent that a standing pressure wave is generated in the cell under the application of a pulse-train signal, and the standing pressure wave generates an array of concentric defects. weak bending deformation with the flow, symmetric or asymmetric splay deformations can appear. However, the field-induced symmetric splay can only be achieved via a first-order transition from the initial weak bend deformation, and the asymmetric splay deformation has a higher elastic deformation energy compared to the twist deformations. Hence, the twist deformations with a director normal to the flow direction are obtained under the application of a vertical electric field and horizontal flow field. Meanwhile, we did not find any evidence of 
a

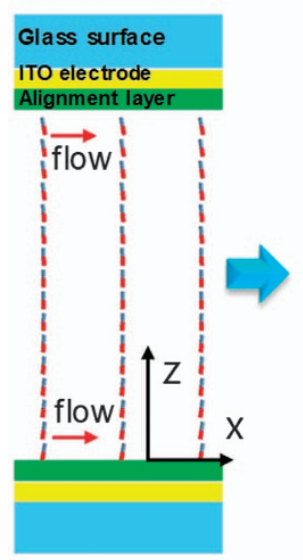

C
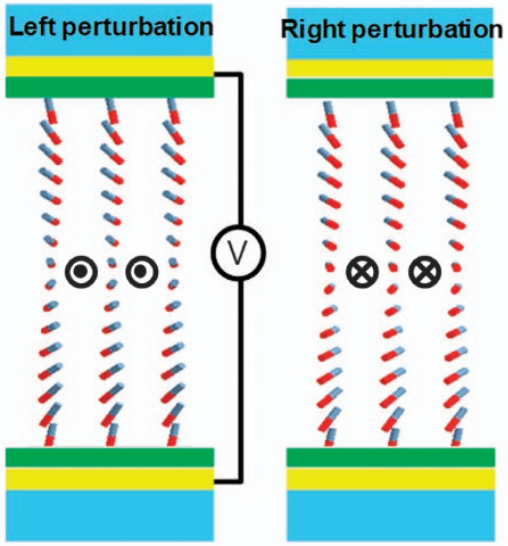

b

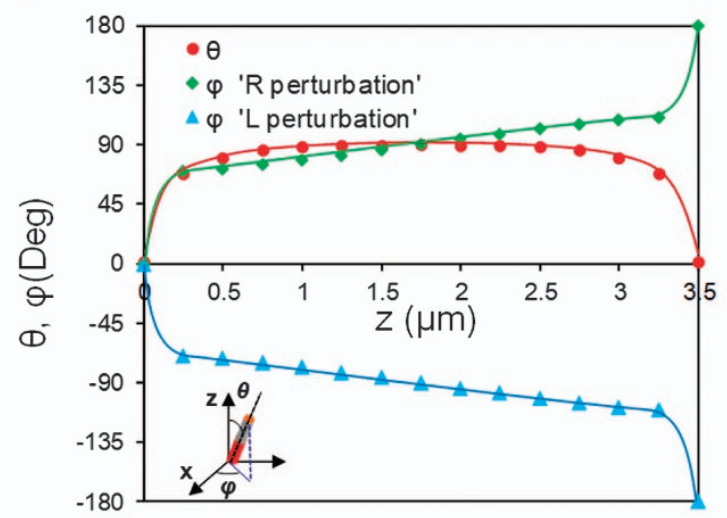

d

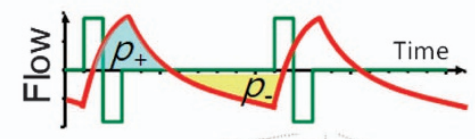

Splay deformation
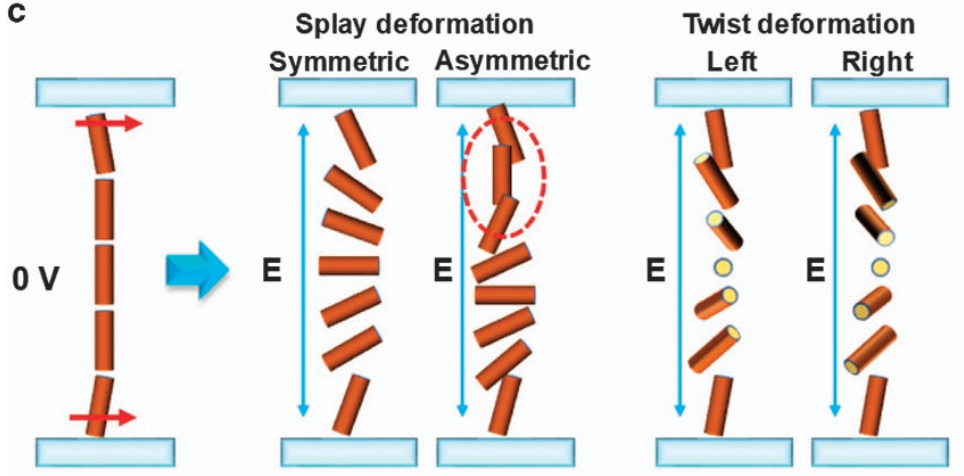

Figure 5 Simulation of the director profile under a weak bending deformation and vertical fields. (a) Simulation results for the director profile. When an LC flow is induced, the shear effect causes the molecules near the surfaces to tilt along the flow direction. When the electric field is subsequently applied, the director profile changes to one with the minimal elastic deformation energy in either the right- or left-twist deformation (left and right sides). (b) The corresponding director tilting directions for the left- and right-twist deformations. (c) Feasible deformations under the application of electric fields (see the main text). (d) The 'pulse ON' signal drives the $p_{+}$pressure wave, which exhibits a pressure maxima in the central region, and the elastic restoring force drives the $p_{-}$pressure wave. Due to the periodic pressure wave, the concentric defect array pattern is obtained.

substrate vibrations in either the Michelson interferometry analysis or the immersion test of a cell in viscous silicon oil (Supplementary Figure 5). These tests confirmed that the periodic director fields are induced by the longitudinal, internal standing pressure waves in the LC layer without the aid of substrate vibrations.

The collective director vibration caused by the pulse-train signal (Figure 3) results in LC flow that is parallel to the substrate, that is, backflow. The standing pressure wave is supposed to be induced by the backflow. The backflows near the top and bottom substrates occur along the opposite directions in a single-domain homeotropic cell. However, when the director has a twist or bend deformation, the backflows near the two surfaces are not opposite and can have a nonzero net flow in a certain direction. ${ }^{28,29}$ The molecular falling and rising motions of the pulse-train signal induce periodic backward and forward backflows parallel to the substrate, generating a vibrational pressure wave. Since the backflow is highly dampened and only exists under the application of external driving forces, the stationary LC molecules outside the electrode edge act as a rigid boundary to reflect the pressure wave. The interference between the backflow wave and reflected wave creates the periodic standing wave. Concentric texture patterns were observed in a square cell with circular electrodes, which also indicated the interference of the backflow and the electrode boundary (Supplementary Figure 6). One should note that the standing pressure wave in the cell is a type of mechanical wave and not an acoustic wave.

The mean director field in Figure 2c was empirically determined, and the director field in the middle layer of the cell can be expressed as:

$$
\begin{aligned}
& \mathbf{n}(x, y, z) \\
& =\left(\frac{\sin (\pi m y) \sin (\Theta(t))}{\sqrt{\sin ^{2}(\pi m x)+\sin ^{2}(\pi m y)}}, \frac{-\sin (\pi m x) \sin (\Theta(t))}{\sqrt{\sin ^{2}(\pi m y)+\sin ^{2}(\pi m x)}}, \cos (\Theta(t))\right),
\end{aligned}
$$

where $m$ is the standing wave mode, and $\Theta(t)$ is the polar angle of the vibrating director according to the pulse signals. The corresponding standing pressure waves, $p(x, y, t)$, and the flow vector fields, $\mathbf{u}(x, y, t)$, in the $x-y$ plane can be expressed as:

$$
\begin{aligned}
& p(x, y, t)=-A\left\{\cos \left(\frac{\pi m x}{a}\right)+\cos \left(\frac{\pi m y}{a}\right)\right\} H(f, t), \\
& \mathbf{u}(x, y, t)=\frac{A}{z_{0}}\left\{\sin \left(\frac{\pi m x}{a}\right) \hat{\mathbf{x}}+\sin \left(\frac{\pi m y}{a}\right) \hat{\mathbf{y}}\right\} H(f, t),
\end{aligned}
$$

Where $A, f, a$ and $z_{0}$ are the amplitude, applied frequency, width of the 

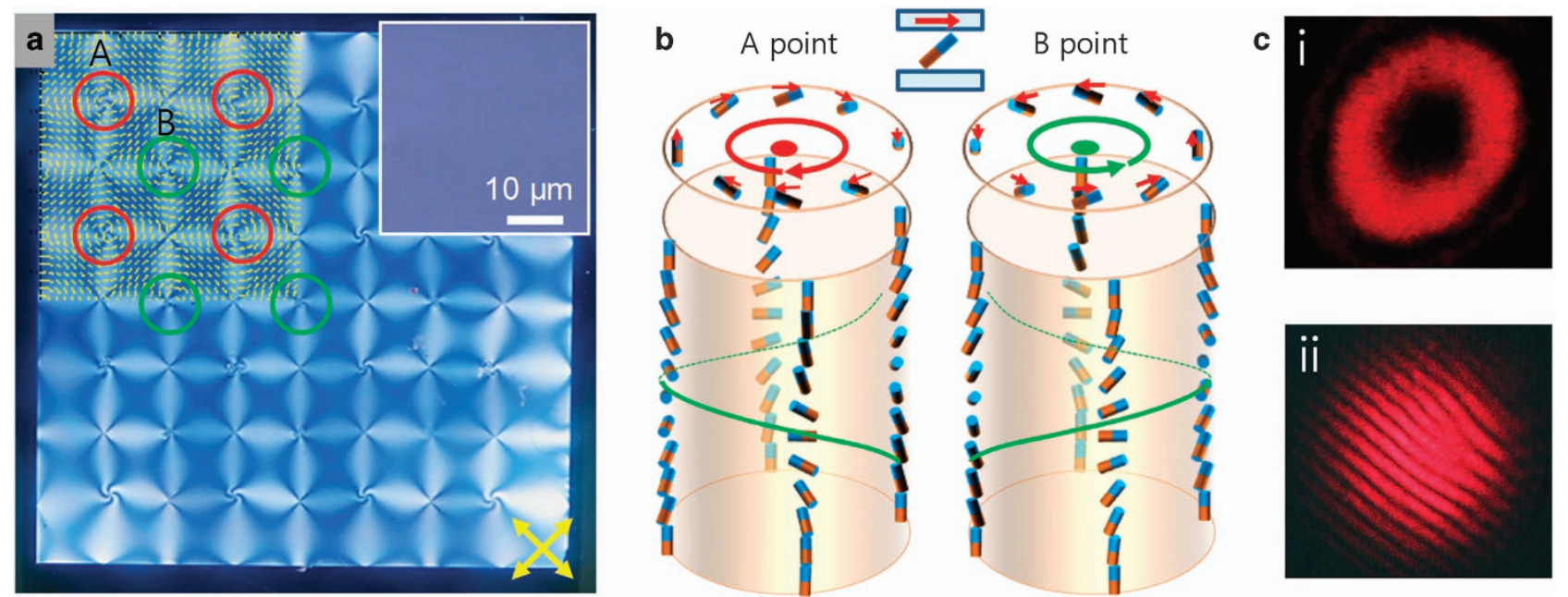

Figure 6 Uniform defect array with spiral director profiles and optical vortex inducer. (a) Adding the R811 chiral dopant ( 0.2 wt\%) eliminated the microdomains, and then, the periodic uniform textures persisted without the presence of a standing wave after switching from a pulse-train signal to a normal square-wave signal (see Supplementary Video 1). A microscopic image reveals no texture (inset). (b) Director profiles around the two defects with oppositehanded spiral structures corresponding to points A and B. (c) The luminance profile of the circular, polarized laser beam became a donut shape after passing through a LC vortex (i), and the interference pattern of the donut-shape beam with another linear polarized light is a fork-shaped interference pattern with two dislocations (ii). This confirms the defect point can serve as an optical vortex inducer.

LC layer and characteristic impedance factor of the medium, respectively, and $H(f, t)$ is the temporal harmonic term. The director fields profile and the flow vector fields in Figures $2 \mathrm{c}$ and $4 \mathrm{c}$ are consistent with the flow-induced reorientation scheme and the total free energy simulation in Figure 5. The flow vectors periodically change their signs, corresponding to the ON and OFF times of the pulse signals (top panel in Figure $5 \mathrm{~d}$ ). The $p^{+}$and $p^{-}$pressure waves in Figure $5 \mathrm{~d}$ are driven by the electric force upon molecular falling and elastic restoring force upon molecular rising, respectively. This causes the asymmetric flow and pressures in the $p_{+}$and $p_{-}$stages, but the mean flow is stationary. Hence, $H(f, t)$ contains higher-order harmonic terms as well, utilizing asymmetric harmonic vibration. The red and green circles in Figure $4 \mathrm{c}$ represent the lowest and highest pressure points in the $p_{+}$stage, respectively, which was experimentally confirmed using a cell containing a chiral dopant.

\section{A uniform defect array with a spiral director profile and optical vortex inducer}

As described in the previous section, the simulation revealed that the left- and right-twist deformations had the same free energy, which resulted in the formation of microdomains at low frequencies. At high frequencies, the consecutive pulse signals were delivered before the molecules recovered their vertical alignment. Hence, the microdomains persisted instead of being refreshed, and they interacted with each other and merged to form macroscopic domains to reduce the elastic deformation energy along the domain boundaries (Figure 2b). By further increasing the frequency $(>160 \mathrm{~Hz})$, the director falling and rising vibrations weakened owing to the slow response of the LCs, which destroyed the standing wave and the patterns because of the instability of the domain boundaries with a high elastic deformation energy. The destruction of the patterns was also observed when the pulse-train signals were replaced by square-wave signals; the abrupt change in the pulse-train signals to a square-wave signal stopped the director vibration and destroyed the defect array patterns (the left cell in Supplementary Video 1). These observations confirmed that the director vibration triggers the generation of the periodic defect array patterns.
By adding a chiral dopant, we were able to obtain uniform defect arrays without microdomains. In the cell containing the chiral dopant, abruptly changing the stimuli pattern from a train of pulses to square waves froze the periodic domains, allowing stationary periodic defect arrays with left- and right-twisting helicities to be obtained (Figure 6a, and the right cell in Supplementary Video 1). The inset microscopic image in Figure 6a shows a uniform LC alignment without any domains. The director profiles around the left- and right-twisting helicities are described in Figure 6b. The arrows on the top circle panel indicate the mean tilting direction of the directors from atop view (see the inset illustration). The combination of the concentric director alignment and the molecular tilting direction creates a spiral alignment of LC directors in the middle region of the cell (green lines in Figure 6b). The spiral handedness of each defect point was the opposite in the cell containing the chiral dopant with opposite handedness (see Supplementary Figure 7 for the details). The opposite spiral handedness in the LCs with the enantiomeric chiral dopants was well predicted based on the simulation results in Figure 5.

The director defects can serve as a good, optical vortex inducer; when a circular, polarized light passes through the defect, the beam profile becomes a donut shape (Figures $6 \mathrm{c}-\mathrm{i}$ ). This is a well-known feature of an optical vortex; the phase in the center of the optical vortex is not defined, and its intensity is zero. When linearly polarized light interferes with the donut-shape beam, a fork-shaped interference pattern with two dislocations appears, confirming an optical vortex with a topological charge of two (Figure $6 \mathrm{c}$-ii). ${ }^{16}$

\section{Unique properties of the standing nematodynamic wave and asymmetric particle actuation}

The spontaneous standing wave and molecular rearrangement in the LC cell have several remarkable features that are different from those that characterize 2D-membrane films and rectangular cavities. ${ }^{7,8}$ First, the pitch values of the periodic units depend on the frequency but not on the amplitude of the applied signals. The pitch decreased in a stepwise manner (Figures $7 \mathrm{a}$ and $\mathrm{b}$ ); periodic patterns with evennumbered units $(m \times m, m=4,6,8$ and 10) were sequentially produced as the frequency increased at a fixed voltage (panels A-D 
a
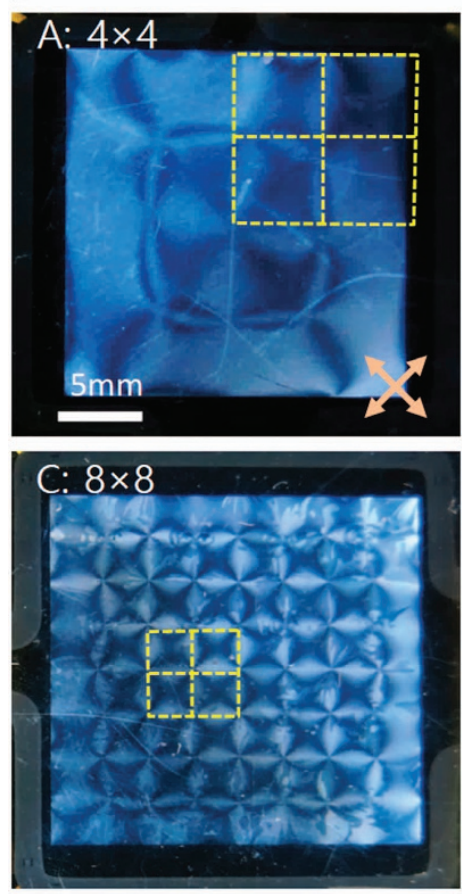
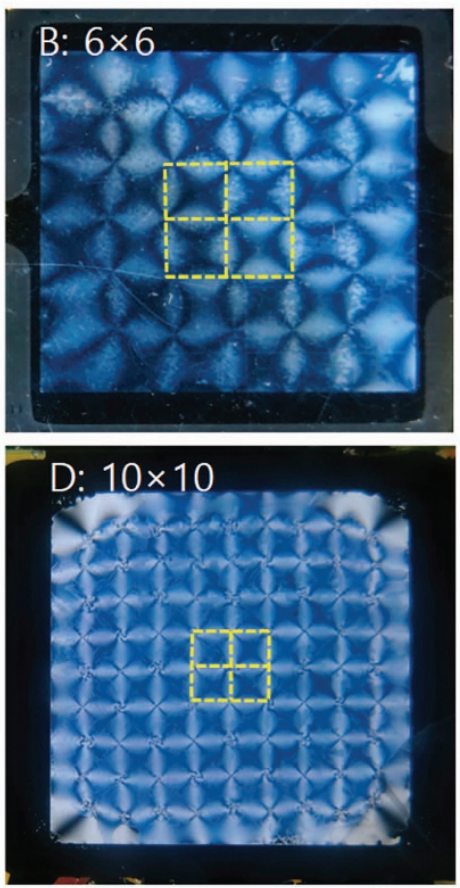

b

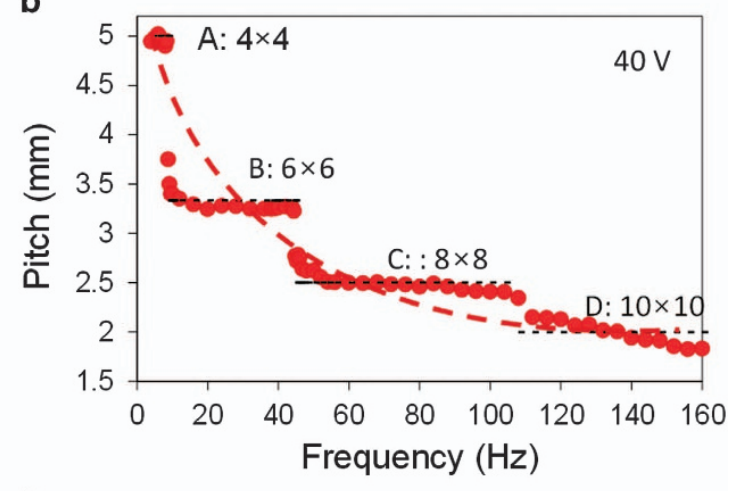

C

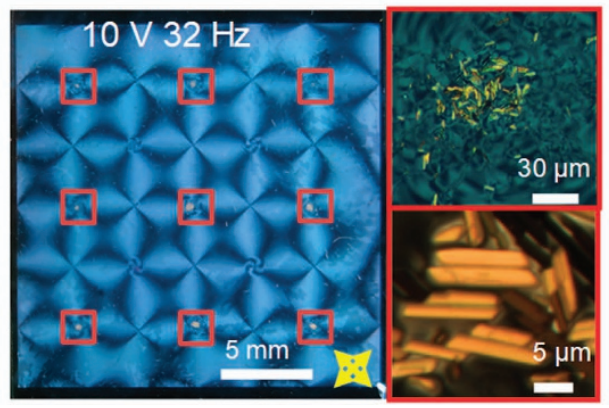

Figure 7 Stepwise reduction in the pitch of the periodic patterns with the increasing frequency. (a) Typical periodic patterns with $n \times n$ units, where $n=4,6$, 8 and 10. (b) The mean pitch of the periodic units as a function of the frequency at which the pulse signals were applied. The mean pitch decreased in a stepwise manner, and each pattern persisted throughout a wide range of frequencies. At frequencies above $120 \mathrm{~Hz}$, the pattern gradually disappeared, starting at the periphery, owing to the decay of the standing wave. (c) C6 micro-rods were gathered at the pressure maxima in the $p_{+}$stage but not at the pressure maxima in the $p_{-}$stage. Expanded images on the right side clearly show the aggregated microparticles.

in Figure 7a). Each pattern was stable, persisted through a wide range of frequencies and transformed into the next mode upon the appearance of additional periodic units with the increasing frequency. This indicates that the wave speed $(v)$ adapts itself to the frequency variation, and the wavelength remains constant $\left(0.5 f \cdot v^{-1}\right)$ within the frequency range of each step. ${ }^{30}$ This is in contrast to conventional standing waves with discrete resonance frequencies. The selfadaptation is supposed to stem from the nonlinear property of the LCs; the acoustic wave velocity in LCs is reported to be highly nonlinear and anisotropic. ${ }^{31-33}$ Second, the standing waves in the LCs, which have diagonal nodal lines and even-numbered modes, are rotated by $45^{\circ}$ compared with the conventional standing waves in other systems that have nodal lines that are parallel to the boundary and integer modes. ${ }^{7,8}$ This unusual geometry is related to the least parallel flux boundary condition (Supplementary Figure 8). Finally, the longitudinal standing pressure wave can asymmetrically transport small particles. ${ }^{34}$ When micro-rods of C6 crystals were added, the C6 rods aggregated at the high-pressure anti-nodes in the $p_{+}$stage during the prolonged application of electric signals, as shown in Figure $7 c$, which confirmed the asymmetric pressure between the $p_{+}$and $p_{-}$ stages. By changing the field frequency, the rods could be relocated to new high-pressure points. It is interesting to note that the particles actuated perpendicular to the mean director direction, which indicated that the particle actuation is not due to the defect-assisted electrophoretic actuation and is due to the standing pressure wave.

\section{DISCUSSION}

We have demonstrated the unique coupling between spontaneous standing nematodynamic waves and director orientation in a nematic
LC cell, and this coupling results from a series of chain interactions: (1) the field-induced director vibration, that is, the periodic falling and rising motion responses to pulse signals, (2) the director vibrationinduced LC flow effect (or backflow), (3) the spontaneous formation of a standing nematodynamic pressure wave due to the coupling between the random LC flow and boundaries, (4) the standing waveinduced rearrangement of the LC director fields and (5) the standing wave stabilization by the nonlinear properties of LCs. We confirmed the coupling between the pressure wave and the director orientation using external mechanical flows and demonstrated the presence of a spontaneous pressure wave via particle actuation. All these observations indicate the nematodynamic standing wave-mediated periodic defect formation. However, a further theoretical study will be needed to clarify the detailed mechanism of the standing nematodynamic wave formation and its abundant new features, such as the selfadaption of the resonance frequency and the diagonal nodal lines. The shape and pitch of periodic patterns are diverse depending on the boundary conditions and the material properties; for example, the periodic pitch may decrease to the micrometer scale when fast response materials, such as ferroelectric LCs, are used. The abundant interesting features of the interaction between the standing wave and molecular ordering may open new vistas in studies on soft matter and optics; in particular, the interaction can be used for fabricating periodic defect arrays with spiral director fields, optical vortex array inducers and micro-lens arrays for optical devices and for studies of particle assemblies.

\section{CONFLICT OF INTEREST}

The authors declare no conflict of interest. 


\section{ACKNOWLEDGEMENTS}

This work was supported by the National Research Foundation of Korea (NRF) grant funded by the Korea government (MSIP) (No. 2014R1A2A1A11054392).

Author contributions: J-KS conceived the project and designed the experiments. LKM conducted all the experiments. Both authors analyzed the data and participated in writing the manuscript.

\section{PUBLISHER'S NOTE}

Springer Nature remains neutral with regard to jurisdictional claims in published maps and institutional affiliations.

1 Barnes, W. L., Dereux, A. \& Ebbesen, T. W. Surface plasmon subwavelength optics. Nature 424, 824-830 (2003).

2 Allison, M., Godfrey, D. A. \& Beebe, R. F. A wave dynamical interpretation of Saturn's polar hexagon. Science 247, 1061-1063 (1990).

3 Čižmár, T., Šiler, M., Šerý, M., Zemánek, P., Garcés-Chávez, V. \& Dholakia, K. Optical sorting and detection of submicrometer objects in a motional standing wave. Phys. Rev. B 74, 035105 (2006).

4 Falkovich, G., Weinberg, A., Denissenko, P. \& Lukaschuk, S. Surface tension: floater clustering in a standing wave. Nature 435, 1045-1046 (2005).

5 Chen, P., Luo, Z., Guven, S., Tasoglu, S., Ganesan, A. V., Weng, A. \& Demirci, U. Microscale assembly directed by liquid-based template. Adv. Mater. 26, 5936-5941 (2014)

6 Courtney, C. R. P., Ong, C. K., Drinkwater, B. W., Bernassau, A. L., Wilcox, P. D. \& Cumming, D. R. S. Manipulation of particles in two dimensions using phase controllable ultrasonic standing waves. Proc. R. Soc. A 468, 337-360 (2011).

7 Sturut, J. W. The Theory of Sound Vol. 1, Ch. IX, 256-259 (Macmillan and Co., London, 1877).

8 Beranek, L. L. Acoustics Ch. 10, 285-289 (The Acoustical Society of America, Woodbury, NY, 1993).

9 Borshch, V., Kim, Y. K., Xiang, J., Gao, M., Jakli, A., Panov, V. P., Vij, J. K., Imrie, C. T., Tamba, M. G., Mehl, G. H. \& Lavrentovich, O. D. Nematic twist-bend phase with nanoscale modulation of molecular orientation. Nat. Commun. 4, 2635 (2013).

10 Yoon, D. K., Choi, M. C., Kim, Y. H., Kim, M. W., Lavrentovich, O. D. \& Jung, H. T. Internal structure visualization and lithographic use of periodic toroidal holes in liquid crystals. Nat. Mater. 6, 866-870 (2007).

11 Tkalec, U., Ravnik, M., Čopar, S., Žumer, S. \& Muševič, I. Reconfigurable knots and links in chiral nematic colloids. Science 333, 62-65 (2011).

12 Richter, H., Buka, A. \& Rehberg, I. Electrohydrodynamic convection in a homeotropically aligned nematic sample. Phys. Rev. E 51, 5886-5890 (1995).

13 Sasaki, Y., Jampani, V. S., Tanaka, C., Sakurai, N., Sakane, S., Le, K. V., Araoka, F. \& Orihara, H. Large-scale self-organization of reconfigurable topological defect networks in nematic liquid crystals. Nat. Commun. 7, 13238 (2016).

14 Son, J. H., Zin, W. C., Takezoe, H. \& Song, J. K. Alignment of liquid crystals using a molecular layer with patterned molecular density. Adv. Mater. 24, 6105-6110 (2012).

15 Kim, S. D., Lee, B., Kang, S. W. \& Song, J. K. Dielectrophoretic manipulation of the mixture of isotropic and nematic liquid. Nat. Commun. 67936 (2015).

16 Barboza, R., Bortolozzo, U., Assanto, G., Vidal-Henriquez, E., Clerc, M. G. \& Residori, S. Vortex induction via anisotropy stabilized light-matter interaction. Phys. Rev. Lett. 109, 143901 (2012).
17 Brasselet, E. Tunable optical vortex arrays from a single nematic topological defect. Phys. Rev. Lett. 108, 087801 (2012).

18 Voloschenko, D. \& Lavrentovich, O. D. Optical vortices generated by dislocations in a cholesteric liquid crystal. Opt. Lett. 25, 317-319 (2000).

19 Brasselet, E., Murazawa, N., Misawa, H. \& Juodkazis, S. Optical vortices from liquid crystal droplets. Phys. Rev. Lett. 103, 103903 (2009).

20 Brasselet, E. Spin-orbit optical cross-phase-modulation. Phys. Rev. A 82 , 063836 (2010).

21 Cai, X., Wang, J., Strain, M. J., Johnson-Morris, B., Zhu, J., Sorel, M., O'Brien, J. L., Thompson, M. G. \& Yu, S. Integrated compact optical vortex beam emitters. Science 338, 363-366 (2012).

$22 \mathrm{Ma}$, X., Pu, M., Li, X., Huang, C., Wang, Y., Pan, W., Zhao, B., Cui, J., Wang, C., Zhao, Z. \& Luo, X. A planar chiral meta-surface for optical vortex generation and focusing. Sci. Rep. 5, 10365 (2015).

23 Guo, J. K. \& Song, J. K. Three-dimensional reconstruction of topological deformation in chiral nematic microspheres using fluorescence confocal polarizing microscopy. Opt. Express 24, 7381-7386 (2016).

24 Shen, T.-Z., Hong, S.-H., Lee, B. \& Song, J.-K. Bottom-up and top-down manipulations for multi-order photonic crystallinity in a graphene-oxide colloid. NPG Asia Mater. 8, e296 (2016).

25 Kim, Y. J. \& Patel, J. S. Acoustic generation in liquid crystals. Appl. Phys. Lett. 75, 1985-1987 (1999).

26 Helfrich, W. Orienting action of sound on nematic liquid crystals. Phys. Rev. Lett. 29, 1583-1586 (1972)

27 Dion, J. L. \& Jacob, A. D. A new hypothesis on ultrasonic interaction with nematic liquid crystal. Appl. Phys. Lett. 31, 490 (1977).

28 Chen, S.-H. \& Yang, C.-L. Dynamics of twisted nematic liquid crystal pi-cells. Appl. Phys. Lett. 80, 3721-3723 (2002).

29 Mieda, Y. \& Furutani, K. Two-dimensional micromanipulation using liquid crystals. Appl. Phys. Lett. 86, 101901 (2005).

30 Boudaoud, A., Couder, Y. \& Ben Amar, M. Self-adaptation in vibrating soap films. Phys. Rev. Lett. 82, 3847-3850 (1999).

31 Virga, E. Variational theory for nematoacoustics. Phys. Rev. E 80, 031705 (2009).

32 De Matteis, G. \& Virga, E. G. Director libration in nematoacoustics. Phys. Rev. E 83, 011703 (2011).

33 Selinger, J. V., Spector, M. S., Greanya, V. A., Weslowski, B. T., Shenoy, D. K. \& Shashidhar, R. Acoustic realignment of nematic liquid crystals. Phys. Rev. E 66, 051708 (2002).

34 Tian, L., Martin, N., Bassindale, P. G., Patil, A. J., Li, M., Barnes, A., Drinkwater, B. W. \& Mann, S. Spontaneous assembly of chemically encoded two-dimensional coacervate droplet arrays by acoustic wave patterning. Nat. Commun. 7, 13068 (2016).

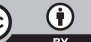

This work is licensed under a Creative Commons Attribution 4.0 International License. The images or other third party material in this article are included in the article's Creative Commons license, unless indicated otherwise in the credit line; if the material is not included under the Creative Commons license, users will need to obtain permission from the license holder to reproduce the material. To view a copy of this license, visit http:// creativecommons.org/licenses/by/4.0/

(C) The Author(s) 2018

Supplementary Information accompanies the paper on the NPG Asia Materials website (http://www.nature.com/am) 